www.nature.com/clinicalpractice/neuro

during an inpatient rehabilitation stay. In total, 14 patients from the RAGT group and 15 patients from the CWT group completed the full course of walking training.

Significant improvements over baseline in $20 \mathrm{~m}$ walking velocity were seen in both the RAGT group and the CWT group $(P=0.003$ and $P=0.026$, respectively). By contrast, only the patients in the RAGT group showed significant improvements in walking distance, and in extensor strength in the right and left knees $(P=0.006, P=0.006$ and $P=0.004$, respectively). Between-group differences as measured by effect size were large for the end points of walking velocity and knee-extensor strength (effect size $>0.6$ for each) and moderate for 6 min walking distance (effect size 0.4-0.6), all favoring RAGT. Walking safety as rated by the patients (Visual Analogue Scale) was lower after RAGT than after CWT, but this difference was not significant. Overall patient satisfaction with RAGT was high. The researchers conclude that larger studies of RAGT in MS are warranted.

Original article Beer S et al. (2007) Robot-assisted gait training in multiple sclerosis: a pilot randomized trial. Mult Scler [doi:10.1177/1352458507082358]

\section{Glutamatergic synapses undergo remodeling during AD progression}

Synaptic density is emerging as a factor that correlates closely with cognitive status in Alzheimer's disease (AD). Glutamatergic synapses in the cortex and hippocampus might be particularly important in the pathology of AD. Bell et al. have now shown that these synapses undergo substantial remodeling during $A D$ progression, including an unexpected upregulation of glutamatergic presynaptic bouton density in patients with mild cognitive impairment.

The authors investigated whether specific markers for glutamatergic neurons can provide more information than traditional neurochemical investigations about the glutamatergic pathology associated with AD. Antibodies highly specific for the vesicular glutamate transporter 1 were used to identify glutamatergic presynaptic elements (boutons) and glutamatergic dystrophic neurites in post mortem midfrontal gyrus brain tissue from participants of the Religious Orders Study, a longitudinal study of aging and AD.

$A D$ pathology was shown to include the generation of glutamatergic dystrophic neurites, which reduces synaptic connectivity, thereby contributing to cognitive decline. As expected, glutamatergic presynaptic boutons were significantly depleted in samples from men and women with mild and severe AD, compared with controls. Increased glutamatergic presynaptic bouton density in each group correlated with improved cognition, except in individuals with mild cognitive impairment. Paradoxically, glutamatergic presynaptic bouton density was elevated in the patients with mild cognitive impairment; the density then dropped as the patients progressed to AD.

The authors conclude that compensatory upregulation of cortical terminals might occur during the mild cognitive impairment that is prodromic of $A D$. They suggest that this period represents a potential therapeutic window within which some of the subsequent neuronal damage that occurs during $A D$ progression can be delayed.

Original article Bell KFS et al. (2007) Paradoxical upregulation of glutamatergic presynaptic boutons during mild cognitive impairment. J Neurosci 27: 10810-10817 